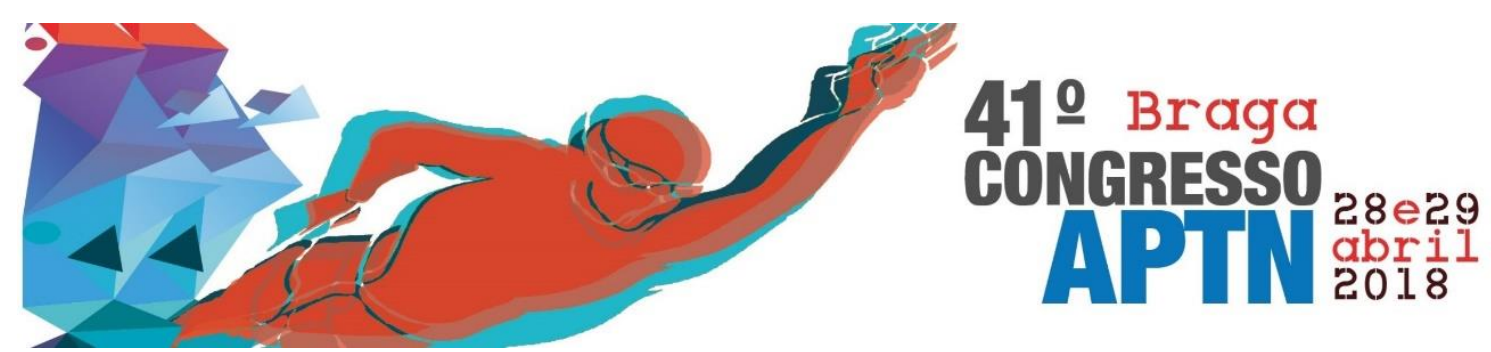

\title{
41 Congresso Técnico e Científico da Associação Portuguesa de Técnicos de Natação - APTN
}

28 e 29 de abril de 2018

Os trabalhos publicados no presente suplemento foram submetidos à apreciação da Comissão Científica do $41^{\circ}$ Congresso Técnico e Científico da Associação Portuguesa de Técnicos de Natação - APTN realizado nos dias 28 e 29 de abril de 2018 no Município de Braga. O conteúdo dos artigos é única e exclusivamente da responsabilidade dos seus autores. A Comissão Científica da APTN não assume qualquer tipo de responsabilidade pelas opiniões e afirmações expressas pelos autores. É permitida a reprodução parcial dos textos e sua utilização sem fins comerciais, desde que devidamente citada a fonte/referência. 


\section{Influência da distância do arco à parede na aquisição da técnica do salto em crianças no processo de adaptação ao meio aquático}

Ana Cabrita ${ }^{1}$, Andreia Ferrum ${ }^{1}$, Carolina Matos ${ }^{1}$, Dmytro Martynenko', Rafael Meloㄹ, Miguel Fortunato ${ }^{1}$, Ana Conceição ${ }^{1}$, David Catela ${ }^{1}$, Marta Martins ${ }^{1}$, Marco Branco ${ }^{1}$

1. Escola Superior de Desporto de Rio Maior, Instituto Politécnico de Santarém, Rio Maior, Portugal

\section{INTRODUÇÃo}

A distância a que o arco se encontra do bordo lateral da piscina pode ser considerada uma variável importante a ter em conta ao quando se aplicam exercícios deste género pois para se estudar a tomada de decisão do desportista, é fundamental atender-se à estrutura do contexto, ao objetivo da tarefa e à dinâmica da interação indivíduo/ambiente (Araújo, Davids, \& Hristovski 2006).

MÉTODos
A amostra foi composta por 12 crianças
$(\mathrm{N}=12), 7$ masculino e 5 feminino, ( 8,75 anos
$\pm 1,30)$ em fase de adaptação ao meio aquático e
sem experiência prévia. A tarefa consistiu em
executar um salto, partindo da posição
engrupada, com os dois pés apoiados no bordo da
piscina, passando por dentro de um arco (com um
diâmetro de $60 \mathrm{~cm})$, colocado na posição vertical.
A cada salto, a distância aumentou (de $10 \mathrm{~cm}$ em
10cm) até que cada elemento da amostra não
conseguisse realizar a tarefa sem que nenhuma
parte do seu corpo entrasse em contacto com o
arco.
Foi efetuado um teste de impulsão vertical. Os
instrumentos utilizados para a medição dos saltos

foram uma fita métrica ajustada às condições (salto de impulsão vertical) e uma tabela de observação construída e validada por peritos (salto através do arco).

\section{RESULTADOS}

De notar melhorias na técnica de execução do salto à medida que a distância do arco à parede aumentava por parte de $75 \%$ dos alunos. À medida que $\mathrm{o}$ arco se afastava da parede as crianças apresentavam comportamentos distintos a cada salto para dentro do arco.

\section{CONCLUSÃO}

A relação entre a distância do arco e a Altura do salto de impulsão vertical é estatisticamente significativa $(p=0,030<0,05)$. O estudo indica que existe uma distância ideal e particular adotada por cada uma das crianças, que varia com a força de impulsão vertical das mesmas.

\section{REFERÊNCIAS}

Araújo, D., Davids, K., \& Hristovski, R. (2006). The ecological dynamics of decision making in sport. Psychology of Sport and Exercise, 7(6), 653-676. https://doi.org/10.1016/j.psychsport.2006.07.00 2

\section{A dynamometric central for $3 \mathrm{~d}$ forces and moments assessment in swimming starts}

Daria Rudnik ${ }^{1,2}$, Karla de Jesus $2,3,7$, Luís Mourão ${ }^{2,3,4}$, Hélio Roesler $2,3,5$, Kelly de Jesus $2,3,7$, Leandro Machado ${ }^{2,3}$, Susana Soares ${ }^{2,3}$, Ricardo J. Fernandes ${ }^{2,3}$, Mário Vaz ${ }^{3,6}$, Marek Rejman ${ }^{1}$, João Paulo Vilas-Boas 2,3

1. School of Physical Education, University of Wroclaw, Poland; 2. CIFI2D, Faculty of Sport, University of Porto, Portugal; 3. LABIOMEP, University of Porto, Portugal; 4. Polytechnic Institute of Porto, Portugal; 5. ESEF, University of the State of Santa Catarina, UDESC, Brazil; 6. LOME, INEGI, Faculty of Engineering, University of Porto, Portugal; 7. FEFF, Federal University of Amazonas, UFAM, Brazil

\section{INTRODUCTION}

Over the years, swimming start technique has evolved in close relationship with the modifications in the swimming rules and technologies developed to assist the swimmers action and to evaluate its proficiency, inspiring new research directions (Vantorre et al., 2010). As sprint swimming events classifications can be decided by very small margins (e.g. $0.01 \mathrm{~s}$ ), sport engineers and biomechanists aimed optimizing instrumented starting blocks for more/multiconditions analysis purposes (de Jesus et al., 
2014). The noticeable technological advances provided by these devices supply swimmers and coaches with new opportunities for assessment and training of technical elements of swimming race as starts and turns. The purpose of this study was to reveal the value of using an instrumented starting block (3D dynamometric central), developed at the University of Porto, as a tool to diverse analysis purpose. Moreover, this review aims describing running research trends in assessment and training in swimming starts and turns optimisation.

\section{METHODS}

To upgrade knowledge in swimming starts and turns researches, this study reviews literature from swimming specific publication, proceedings of scientific conferences and academic thesis $(\mathrm{PhD})$

\section{RESULTS}

The design and construction of a 3D force plate prototype, which might be use as a modular sensor of an instrumented starting block, was described by Mourao et al. (2016). The linear static and model simulations shown that the dynamometric central might be used as a tool for starts and turns detailed analysis (Vilas-Boas et al. 2014). Evaluation of value in use was performed during a large number of data acquisitions taking place in Porto University under the patronage of LABIOMEP, the University of Porto Biomechanics Laboratory. These experiments provided results proving that swimming start and turns performance evaluations are an invaluable tool for technical optimisation for all level swimmers. Different studies were conducted, including biomechanical analysis of starts and turns, considering different positioning of upper and lower limbs on starting block and its configuration on changes in performance, comparison to results from land tests, as well as analysis of dynamical asymmetry. Moreover, the high sensitivity of the device allows specific physical analysis of swimmers actions.

\section{CONCLUSIONS}

The Portuguese 3D dynamometric central could be considered as the most modern and versatile instrumented starting block complying with the FINA facilities and starting rules, allowing independent measurement of external forces and moments performed by each limb, independently, at ventral and dorsal starting and turning techniques.

\section{Funding}

Projeto Metaheurísticas da computação natural aplicadas à biomecânica das partidas e viragens em natação (Programa CAPES/FCT Edital 039/2014).

\section{REFERENCES}

de Jesus et al. (2014). The backstroke swimming start: state of the art. Journal of Human Kinetics, 42(1), 27-40.

Mourão et al. (2016). Design and construction of a 3D force plate prototype for developing an instrumented swimming start block. Journal of Biomedical Engineering and Informatics, 2(2), 99.

Vantorre et al. (2010). Kinematical profiling of the front crawl start. International Journal of Sports Medicine, 31(01), 16-21.

Vilas-Boas et al. (2014) A novel dynamometric central for $3 D$ forces and moments assessment in swimming starting. XII Symposium on Biomechanics and Medicine in Swimming. Australian Institute of Sport, 135-136.

\section{Proposta de programa de exercício aquático para indivíduos com osteoartrose}

\section{Fátima Ramalho ${ }^{1}$, Susana Franco ${ }^{1}$, Filipa Maia ${ }^{1}$, Pedro Morouço ${ }^{2}$}

1. Escola Superior de Desporto de Rio Maior, Instituto Politécnico de Santarém, Rio Maior, Portugal; 2. Centro para o Desenvolvimento Rápido e Sustentado de Produto, Instituto Politécnico de Leiria, Marinha Grande, Portugal

\section{INTRODUÇÃO}

As condições demográficas que a sociedade vem assistindo, acarretam problemas de natureza diversa. Como uma das principais preocupações para a saúde pública, surge uma elevada associação entre o envelhecimento e a obesidade, deficiências nutricionais e a (in)atividade física. Assim, doenças relacionadas com a cartilagem 
estão na lista das principais preocupações da Organização Mundial de Saúde, assumindo-se a prevenção da degeneração da cartilagem articular como uma questão importante para a qual existem poucas soluções eficazes. Torna-se premente encontrar estratégias preventivas, que consigam promover uma diminuição da incidência de osteoartrose crónica. Pretende-se com esta proposta, avançar com uma metodologia própria que incida sobre a aplicação do exercício físico em meio aquático, tendo em consideração as limitações dos indivíduos com osteoartrose.

\section{DESENVOLVIMENTO}

A presente proposta foi desenvolvida tendo em conta a abordagem de uma intervenção complexa, com recurso a: (i) literatura; (ii) Fisiologistas do Exercício com especialização em hidroginástica; (iii) na intervenção com praticantes com diagnóstico de osteoartrose; e (iv) profissionais de saúde. O programa assenta em sessões de treino com a duração de 30 a 45 min, 3 vezes por semana. Tirando proveito das propriedades físicas da água, é definido uma utilização gradual da extensão das alavancas, como forma de incremento da carga. O aquecimento deverá incidir sobre a consciencialização do alinhamento corporal, mobilidade articular e respiração. A parte fundamental assenta em exercícios que combinam a componente cardiorrespiratória com a componente de força, promovendo uma amplitude articular progressiva. Na parte final de cada sessão existe uma diminuição progressiva da carga, alternando segmentos corporais. Decorrente das esperadas adaptações, é proposto um programa de 12 semanas, subdividido em 3 partes de 4 semanas cada.

\section{CONCLUSÃO}

A proposta apresentada pretende ser uma ferramenta para implementação de um comportamento saudável, com base num programa de prática de exercício físico para prevenir a osteoartrose. Com uma tendência demográfica para o envelhecimento da população, hoje em dia a sociedade arrastou-se para um ciclo vicioso relativamente à relação comprovada entre a osteoartrose e a obesidade, e o aumento da prevalência de ambos. Para contribuir para a resolução destes problemas é obrigatório ter perspetivas e abordagens de intervenção integradas e multidisciplinares, que promovam uma atividade motivante e duradoura.

Financiamento:

Este trabalho é enquadrado no projeto POCI/01/0145/FEDER/023423 cofinanciado pelo Programa Operacional de Competitividade e Internacionalização (COMPETE 2020), no âmbito do Programa Portugal 2020, apoiado pelo FEDER - Fundo Europeu de Desenvolvimento Regional, da União Europeia.

\section{A exposição ao ruído ocupacional em piscinas cobertas}

Filipe Teixeira ${ }^{1}$

1. M. Eng. Humana, Lic Educ Física e Desporto

\section{INTRODUÇÃo}

A exposição a níveis de ruído superiores a 80 e $90 \mathrm{~dB}(\mathrm{~A})$ envolve determinado grau de risco. A sistémica exposição acima dos valores de conforto e considerados elevados, provocam o incremento do risco de perda de audição, aumento do stress, de patologias cardiovasculares, entre outras relacionadas com a saúde do indivíduo bem como diminui o desempenho na produtividade (Chang et al., 2011). Os profissionais da área da Educação Física e Desporto (Treinadores, Professores, Monitores e Personal Trainers) que trabalham em piscinas cobertas estão expostos ao ruído que é oriundo de várias fontes, desde o gerado pelo movimento da água até à música que é utilizada nas aulas, passando pelos apitos no controlo das aulas ou a verbal para transmissão de informação necessária aos alunos. Estes sons quando conjugados em simultâneo poderão estimular valores de pressão sonora elevados. A agravar toda esta situação, as piscinas cobertas são áreas onde as condições acústicas são naturalmente inadequadas, motivado pela inexistência de materiais de absorção possibilitando a redução de ruído. O resultado traduz-se numa exposição dos professores/treinadores (Prf/Trn) ao ruído e, consequentemente, ao possível desenvolvimento de perdas auditivas (Maffei et al., 2009). MÉTODOS: O estudo desenvolveu-se no sentido de avaliar em contexto de aula o ruído, na procura de perceber qual a exposição ao ruído dos Prf/Trn e demais utilizadores, bem como tentar encontrar processos de minimizar o ruído nestes espaços. A 
investigação realizou-se no norte de Portugal, nos meses de Março e Abril (2014) em piscinas cobertas $(n=4)$ em contexto de aula. O processo de selecção das piscinas baseou-se na localização, nas características arquitectónicas e nas realidades de funcionamento $\left(\mathrm{n}^{\mathrm{O}}\right.$ de colaboradores, $\mathrm{n}^{\circ}$ de utentes, e ocupação). A pesquisa foi organizada em dois estádios, a elaboração, validação e aplicação de um questionário aos Prf/Trn $(n=61)$ e a medição dos níveis de pressão sonora nas piscinas e tratamento estatístico dos dados recolhidos. Utilizou-se o sonómetro com uma precisão de \pm 1 $\mathrm{dB}$, com filtro de oitavas para medir a pressão sonora. No tratamento estatístico utilizou-se o IBM ${ }^{\circledR}$ SPSS ${ }^{\circledR}$ Statistics versão 22.0. RESULTADOS: Os resultados indicaram que 93,4\% dos Prf/Trn estão expostos a níveis de pressão sonora muito elevados mais de 2 horas e que $34,4 \%$ está mais de 6 horas expostos a esses níveis. Os níveis de pressão sonora variaram entre os $75,5 \mathrm{~dB}(\mathrm{~A})$ e os $101,2 \mathrm{~dB}(\mathrm{~A})$, relativamente aos valores de LEX,8h médio semanal situaramse entre os $83 \mathrm{~dB}(\mathrm{~A})$ e os $90 \mathrm{~dB}(\mathrm{~A})$. Tendo-se apurado que os Prf/Trn (100\%) têm noção da presença de ruído nas piscinas, verificou-se que a maioria dos Prf/Trn não é sensível ao ruído $(52,5 \%)$ e que $30 \%$ indicaram que o ruído tem influência no seu desempenho, obrigando a alterar a dinâmica da aula/treino. DISCUSSÃO:
As indicações não são animadoras, os níveis de pressão sonora nas piscinas cobertas são muito elevados, acima dos valores de acção e limite definidos na legislação portuguesa, podendo os Prf/Trn e utilizadores padecer de prejuízos na saúde. O comportamento dos Prf/Trn quanto à exposição ao ruído, não parece ser orientado no sentido da diminuição da sua exposição, pois não procuram reduzir nem se proteger para o efeito. Os gestores das piscinas não apresentam preocupação no controlo do ruído e da sua exposição, não existem quaisquer orientações limitativas quanto à exposição dos Prf/Trn nem quanto às fontes de ruído. Como conclusão podese considerar que as piscinas cobertas como ambientes doentios, quanto ao ruído. Os Prf/Trn na maioria não são sensíveis ao ruído e têm uma atitude negligente quanto à sua exposição.

\section{REFERÊNCIAS:}

Chang, T.-Y., Liu, C.-S., Huang, K.-H., Chen, R.-Y., Lai, J.-S., \& Bao, B.-Y. (2011). High-frequency hearing loss, occupational noise exposure and hypertension: a cross-sectional study in male workers. Environmental Health: A Global Access Science Source, $10, \quad 35$. https://doi.org/10.1186/1476-069X-10-35

Maffei, L., Iannace, G., Masullo, M., \& Nataletti, P. (2009). Noise exposure in school gymnasia and swimming pools. Noise Control Engeenering, 57(6), 603-612.

\section{A importância da interação médico/treinador no "ombro do nadador"}

Luís Duarte Silva1,2, Renato Andrade ${ }^{1,2}$, Alberto Monteiro ${ }^{1,2}$, Isabel Lopes ${ }^{1,2}$, Rogério Pereira ${ }^{1,2}$, Nuno Sevivas ${ }^{1,2}$, Ricardo Bastos ${ }^{1,2}$, João Espregueira-Mendes ${ }^{1,2}$

1. Clínica do Dragão, Espregueira-Mendes Sports Centre - FIFA Medical Centre of Excellence (Porto, Portugal), 2. Dom Henrique Research Centre (Porto, Portugal)

\section{INTRODUÇÃO}

A excelência competitiva requer uma abordagem multidisciplinar na carreira do nadador, onde o papel do treinador permanece fulcral. No entanto, o papel de outros intervenientes, como é o caso do médico sensibilizado para a patologia do nadador, tem sido relegado para papeis secundários, sendo este normalmente restringido ao exame médico desportivo no início de época e à intervenção no momento de lesão. Por este motivo, é da maior importância a interação entre corpo clínico e a restante equipa técnica no sentido de se otimizarem estratégias ao nível da prevenção, correto diagnóstico e tratamento de lesões no nadador. Neste sentido, os autores consideram de extrema importância a divulgação e promoção do trabalho dos prestadores de saúde no atleta nadador.

\section{DESENVOLVIMENTO}

O termo "ombro do nadador" está associado às lesões de repetição (overuse) podendo, de uma forma geral, incluir o conflito subacromial, a tendinopatia da coifa dos rotadores e da longa porção do bicipete. Embora não exista evidencia para todos os fatores de risco de lesão no ombro dos nadadores propostos na literatura, os mais comumente citados incluem: laxidez articular; discinesia escapular; desequilíbrio muscular da 
coifa dos rotadores; nível competitivo; tipo de estilo; volume de treino e utilização de palas, pelo que podemos claramente identificar fatores modificáveis e não modificáveis, quer intrínsecos ou extrínsecos.

Perante o exposto previamente podemos indiscutivelmente afirmar que uma coordenação entre os diversos elementos técnicos é essencial pela própria natureza multifatorial dos fatores de risco modificáveis.

Quanto ao tratamento das afeções do ombro do nadador podemos afirmar na generalidade que a maioria passa por tratamento de medicina física e reabilitação, com ou sem infiltrações intraarticulares na fase inicial, com períodos de reabilitação variáveis consoante a patologia de base e o fator predisponente. Relativamente ao tratamento cirúrgico das principais patologias do ombro do nadador podemos afirmar que o retorno à competição é variável (consoante o tipo de lesão subjacente) podendo ser de apenas poucos meses de ausência após a cirurgia ou de mais de 6 meses. Para além disso, apresenta resultados francamente negativos nomeadamente no que se refere ao tratamento da laxidez articular, com o retorno ao mesmo nível competitivo a rondar apenas os $20 \%$.

Em virtude do tempo de ausência competitivo e a elevada taxa de abandono desportivo após cirurgia, é nossa interpretação que a coordenação multidisciplinar deve ser a base para a prevenção de lesões no ombro do nadador, diminuindo a taxa de incidência de lesão no nadador, tempo de recuperação da lesão sem competição, comorbilidades e impacto socioeconómico associado.

\section{REFERÊNCIAS}

Andrade Mdos, S., et al. (2010). Profile of isokinetic eccentric-to-concentric strength ratios of shoulder rotator muscles in elite female team handball players. $J$ Sports Sci, 28(7), 743-9.

Giotis, D., et al. (2017). Effectiveness of Biologic Factors in Shoulder Disorders. Open Orthop J, 11, 163-182.

\section{Determinação da velocidade crítica de nadadores jovens}

Edgar Ribeiro ${ }^{1}$, Rita Barbosa ${ }^{1}$, Ricardo J. Fernandes 2,3 , Susana Soares ${ }^{2,3}$

1. Faculdade de Desporto, Universidade do Porto, Porto, Portugal; 2. Centro de Investigação, Formação, Inovação e Intervenção em Desporto, Faculdade de Desporto, Universidade do Porto, Porto, Portugal; 3. Laboratório de Biomecânica do Porto, Universidade do Porto, Porto, Portugal.

A determinação da velocidade crítica (VC), a velocidade máxima de nado que se consegue manter por um longo período de tempo sem exaustão (Wakayoshi, 1992), permite prescrever séries de treino ao limiar anaeróbio e analisar a evolução dos nadadores. As distâncias mais recomendadas para determinação da VC implicam uma de curta e outra de longa ( $>15$ min) durações, tornando o processo moroso. Zacca et al. (2016) propuseram os $400 \mathrm{~m}$ como alternativa, obtendo-se a VC multiplicando a V400 por 0.92. O presente estudo pretendeu avaliar a evolução de nadadores jovens em dois macrociclos contíguos usando esta metodologia. Seis nadadores (12.67 \pm 0.52 anos; $48.97 \pm 7.90$ $\mathrm{kg} ; \quad 162 \pm 0.09 \mathrm{~cm})$ e quatro nadadoras $(11.50 \pm 0.58$ anos; $42.55 \pm 8.33 \mathrm{~kg} ; 151 \pm 0.09$ $\mathrm{cm})$ realizaram oito testes de $400 \mathrm{~m}$ durante os primeiro e segundo macrociclos da época desportiva, podendo observar-se os valores da VC na Tabela I. Pode concluir-se que o treino teve um efeito positivo no desenvolvimento da resistência dos nadadores.

Tabela 1.

Valores da VC determinados com o método sugerido por Zacca et al. (2016).

\begin{tabular}{|c|c|c|c|c|c|c|c|c|}
\hline & $\begin{array}{c}\text { T1 } \\
22.09\end{array}$ & $\begin{array}{c}\text { T2 } \\
28.10\end{array}$ & $\begin{array}{c}\text { T3 } \\
18.11\end{array}$ & $\begin{array}{c}\mathrm{T} 4 \\
07.12\end{array}$ & $\begin{array}{c}\text { T5 } \\
06.01\end{array}$ & $\begin{array}{c}\text { T6 } \\
27.01\end{array}$ & $\begin{array}{c}\mathrm{T} 7 \\
14.02\end{array}$ & $\begin{array}{c}\text { T8 } \\
06.03\end{array}$ \\
\hline कా & $1.05 \pm 0.11$ & $1.09 \pm 0.13$ & $1.10 \pm 0.12$ & $1.11 \pm 0.12$ & $1.09 \pm 0.11$ & $1.14 \pm 0.10$ & $1.17 \pm 0.11$ & $1.18 \pm 0.10$ \\
\hline 8 & $1.00 \pm 0.02$ & $1.04 \pm 0.06$ & $1.06 \pm 0.06$ & $1.08 \pm 0.05$ & $1.09 \pm 0.04$ & $1.08 \pm 0.03$ & $1.11 \pm 0.05$ & $1.12 \pm 0.04$ \\
\hline $\mathrm{X} \pm \mathrm{SD}$ & $1.03 \pm 0.08$ & $1.07 \pm 0.10^{*}$ & $1.08 \pm 0.10^{*}$ & $1.10 \pm 0.10^{\mathrm{a}}$ & $1.09 \pm 0.08$ & $1.11 \pm 0.08$ & $1.15 \pm 0.09^{*}$ & $1.16 \pm 0.09^{\mathrm{a}, \mathrm{b}}$ \\
\hline
\end{tabular}

Legenda: T - teste; T1(22.09); T2(28.10); T3(18.11); T4(07.12); T5(06.01); T6(27.01); T7 (14.02); T8(06.03); *Diferente do teste anterior; ${ }^{\mathrm{a} D i f e r e n t e}$ de $\mathrm{T} 1$; ${ }^{\mathrm{b}}$ Diferente de $\mathrm{T} 5$. 


\section{REFERENCES}

Wakayoshi, K.; Ilkuta, K.; Yoshida, T.; Udo, M.; Moritani, T.; Mutoh, Y. (1992). Determination and validity of critical velocity as an index of swimming performance in the competitive swimmer. Eur J Appl Physiol 64:153-157.
Zacca, R.; Fernandes, R. J.; Pyne, D. B., Castro, F. A. (2016). Swimming training assessment: the critical velocity and the 400-m test for age-group swimmers. J Strength Cond Res 30 (5): 13651372 .

\section{Proposta metodológica do treino de hipertrofia em jovens nadadores}

Mariana Cardoso ${ }^{1}$, Miguel Falcão ${ }^{1}$, Pedro Quaresma1, Sara Cruz ${ }^{1}$

1. Universidade de Lisboa, Faculdade Motricidade Humana

\section{INTRODUÇÃO}

O trabalho pretende apresentar uma proposta para o momento ideal, baseado no modelo racional do Long Term Athlete Development, da aplicação do treino de força, mais especificamente da força máxima, utilizando os métodos hipertróficos. Por fim apresentamos as adaptações da carga necessárias para o jovem atleta, concluindo com uma proposta prática, sob a forma de três sessões tipo para dois escalões.

\section{DESENVOLVIMENTO}

O modelo Long Term Athlete Development $(L T A D)$ foi desenvolvido para, de forma planeada, sistemática e gradual acompanhar o desenvolvimento das crianças e jovens na sua vida desportiva. Utilizando o modelo Long Term Athlete Development Strategy (LTAD Strategy), guia do modelo $L T A D$ para a natação, pretendemos entender o que é necessário para que, em cada momento, se consiga trabalhar competências necessárias, de maneira a proporcionar um crescimento e maturação harmonioso nos jovens atletas, não focando apenas em ganhos a curto prazo, mas sim no desenvolvimento efetivo do atleta a longo prazo (Balyi, Way \& Higgs, 2013). O modelo Long Term Athlete Development Strategy, está dividido em sete fases, sendo que cada uma tem as suas caraterísticas principais e tem como objetivo o desenvolvimento dos atletas.

Sendo o treino de força nos jovens de grande importância, é necessário respeitar as janelas de oportunidade de desenvolvimento, onde o organismo tem uma especial sensibilidade e reação positiva aos estímulos exteriores e, onde a carga de treino aplicada nos jovens deve ser especialmente cuidada (Marques, 2010).

O Pico Velocidade e Altura acontece aproximadamente nos 11,4 anos nas raparigas e aos 13,4 anos nos rapazes, e é neste período que, segundo o modelo LTAD Strategy, é feita a abordagem ao treino de força (fases Train to train e Train to compete). Nestas fases o treino de força em jovens nadadores passa por trabalhar, com maior foco, ao nível do ombro, cotovelo, zona abdominal e estabilidade do tornozelo. Seguindo a lógica destes princípios e atendendo aos períodos necessário para a ocorrência de adaptações e respeitando às características específicas dos jovens, uma organização típica da carga para os métodos hipertróficos seria:

Tabela 2

Proposta de treino de força para jovens (Adaptado de Dubig, 2013)

\begin{tabular}{ccc}
\hline Intensidade e Repetições: & 12 a 15 anos & $8-15$ RM $(70 \%$ RM $)$ \\
& 15 a 18 anos & $6-15$ RM $(80 \%$ RM $)$ \\
\hline Séries: & 1 a 3 por grupo muscular \\
Intervalo entre Séries: & 2 minutos \\
Número de Sessões: & 2 a 3 vezes por semana (não consecutivas) \\
\hline
\end{tabular}

\section{REFERÊNCIAS}

Balyi, I., Way, R., \& Higgs, C. (2013). Long-term athlete development. Human Kinetics. Capítulo 1

Duhig, S. Strength training for the young athlete. Journal of Australian Strength Conditioning. 21(4)5-13. 2013
Marques M, (2010). O treino de força em crianças e jovens, Rev Medicina Desp in forma, 1 (6), pp 21 24

Swimming Natation Canada. (2008). Long term athlete development strategy. Ottawa. Publicado em: https://swimming.ca/content/uploads/2015/06/ $\underline{\text { ltad_en.pdf }}$ 


\section{Avaliação das assimetrias propulsivas em nado livre: ferramenta e diagnóstico para o processo de treino.}

Raul F. Bartolomeu ${ }^{1,2}$, Pedro Rodrigues ${ }^{1}$, Jorge E. Morais ${ }^{2,4}$, Daniel A. Marinho ${ }^{3,4}$, Mário J. Costa ${ }^{1,2}$, Tiago M. Barbosa $2,4,5$

1. Instituto Politécnico da Guarda, Guarda, Portugal; 2. Instituto Politécnico de Bragança, Bragança, Portugal; 3. Universidade da Beira Interior, Covilhã, Portugal; 4. Centro de Investigação em Ciências do Desporto, Ciências da Saúde e Desenvolvimento Humano, CIDESD, Portugal; 5. Nanyang Technological University, Singapura

\section{INTRODUÇÃo}

Atualmente já é possível adquirir no mercado sensores de pressão que se colocam nas mãos e/ou pés de onde se derivam as forças propulsivas com a possibilidade de se detetarem possíveis assimetrias. Foi objetivo do presente estudo ter uma compreensão clara de tais assimetrias num ambiente ecologicamente válido (p.e., nado dinâmico em vez de nado amarrado).

\section{MÉTODOS}

Um nadador do sexo masculino com 15 anos, medalhado a nível nacional realizou aleatoriamente três sprints de $25 \mathrm{~m}$ à velocidade máxima em três variantes do nado de crol: completo (C), só com membros superiores (MS) e só com membros inferiores (MI). A medição da força propulsiva em cada uma das condições foi efetuada com recurso a um sistema de monitorização (Aquanex System, DU2V, STR, EUA). Como variáveis definiram-se: o pico de força, a força média, o índice de assimetria (IS) como descrito por Robinson et al (1987), a velocidade média (v) e a frequência gestual (FG).

\section{RESULTADOS}

Os resultados estão apresentados ta tabela 1 .

Tabela 1

Valores obtidos nas três variantes do nado de crol.

\begin{tabular}{|c|c|c|c|c|c|c|c|c|c|c|}
\hline \multirow{2}{*}{\multicolumn{2}{|c|}{ Condição }} & \multicolumn{2}{|c|}{ Pico Força (N) } & \multirow{2}{*}{ IS (\%) } & \multicolumn{2}{|c|}{ Força Média (N) } & \multirow{2}{*}{ IS (\%) } & \multirow{2}{*}{$\mathrm{V}(\mathrm{m} / \mathrm{s})$} & \multicolumn{2}{|c|}{ FG (ciclos/min) } \\
\hline & & E & D & & $\mathrm{E}$ & $\mathrm{D}$ & & & $\mathrm{E}$ & $\mathrm{D}$ \\
\hline \multirow{2}{*}{$\mathrm{C}$} & Braço & $\begin{array}{l}--- \\
\end{array}$ & 162,96 & ---- & ---- & $70,77 \pm 41,32$ & ---- & \multirow{2}{*}{$1,6 \pm 0,13$} & ---- & 60 \\
\hline & Perna & ---- & 121,67 & ---- & ---- & $42,07 \pm 25,79$ & ---- & & ---- & 177 \\
\hline MS & & 139,31 & 129,72 & -7 & $51,77 \pm 44,63$ & $53,27 \pm 39,31$ & 3 & $1,4 \pm 0,12$ & & \\
\hline MI & & 139,35 & 140,18 & 1 & $39,77 \pm 23,05$ & $46,31 \pm 26,87$ & 15 & $0,66 \pm 0,08$ & & \\
\hline
\end{tabular}

\section{DISCUSSÃO}

A única simetria superior a $10 \%$ verificou-se no valor médio de propulsão no nado de pernas, sugerindo-se a necessidade de um maior trabalho ao nível de coordenação e reforço muscular. A maior média de força exercida e a menor frequência gestual na condição MI comparativamente a $\mathrm{C}$, sugere que há um potencial da pernada que não é aproveitado durante o nado completo, apontando a necessidade de treino de coordenação MS/MI para se poder tirar o melhor proveito da propulsão da pernada.

\section{REFERÊNCIAS}

Robinson, R. O., Herzog, W., \& Nigg, B. (1987). Use of force platform variables to quantify the effects of chiropractic manipulation on gait symmetry. Journal of manipulative and physiological therapeutics, $10(4), 172-176$.

\section{Estudo exploratório do perfil psicológico de participação desportiva em atletas de longa distância}

Sebastião Santos ${ }^{1,2}$

1. Universidade de Trás-os-Montes e Alto Douro; 2. Clube de Atletismo de Ferreira do Zêzere

\section{INTRODUÇÃO}

A necessidade de facultar instrumentos simples mas eficazes num mundo cada vez mais tecnológico e rápido, tal como a necessidade de ter os melhores indivíduos e os mais capazes em qualquer equipa, seja ela desportiva ou empresarial. Um maior conhecimento e compreensão do perfil psicológico na regulação dos fatores biopsicosociais dos indivíduos, merece particular atenção, podendo ser crucial para o desenvolvimento e manutenção dos sujeitos nos meios em que estão inseridos. 
Conhecer de forma profunda as variáveis pessoais que distinguem os desportistas nos diferentes perfis psicológicos permitirá a quem intervém no contexto desportivo e empresarial de melhorar a qualidade da sua intervenção. $O$ objetivo do estudo é elaborar e construir um instrumento que defina o perfil psicológico de participação desportiva de atletas de longa distância.

\section{MÉTODOS}

A amostra foi constituída por 34 nadadores dos campeonatos nacionais de longa distância master de uma época desportiva. A média de idades é de 42,2 $(11,1)$ anos e com uma média de experiência de competição de 9,6 $(7,9)$ anos. Procedemos à construção de um instrumento de medida para aferir o perfil psicológico de participação desportiva em atletas de longa distancia. De acordo com os objetivos foi necessário desenvolver e validar um instrumento que define e traça os comportamentos dos atletas de acordo com o tipo de prova, contundente com os desportos onde se desenvolve uma metodologia observacional para uma compreensão mais profunda da realidade que se desenvolve no contexto desportivo (Santos et al., 2014). Na análise das questões foi realizada uma avaliação qualitativa dos dados obtidos, de forma indutiva e dedutiva, tendo sido agrupadas em dimensões de acordo com o seu teor. Foi utilizado uma análise de Cluster em dois grupos.

\section{RESULTADOS}

Verificamos que os dados revelam dois perfis distintos de atletas de acordo com as respostas dadas. Constata-se que os atletas do primeiro grupo são indivíduos que apreciam os momentos em família, gostam de estar com os colegas, de conviver mas sem excessos, são decididos e trabalhadores, têm um sentido de superação nos desafios que surgem, e de orgulho e satisfação quando realizam o que pretendiam, e com uma vontade constante de ultrapassar limites. São indivíduos que se sentem orientados para as provas de longa distância, não apreciam os momentos de espera antes da prova. Se pudessem gostariam de viajar mais e de realizar provas nesses locais.

\section{DISCUSSÃO}

O perfil evidenciado por estes atletas revela indivíduos com uma enorme persistência e vontade de vencer, de ultrapassar e atingir os objetivos definidos e com uma enorme resiliência ao stress. Os indivíduos de certas modalidades são mais resistentes e têm determinadas características psicológicas que lhes permite adaptar-se à dureza de certos desportos (Reigal et al., 2018), tal como é o caso das provas de longa distância. Os sujeitos serão uma mais valia para as instituições onde estão integrados, tendo um autodomínio, no qual o stress é minimizado ou suprimido e a resiliência interna é treinada.

\section{REFERÊNCIAS}

Reigal, D. R., Delgado-Girart, J. E., Raimundi, M. J., \& Hernández-Mendo A. (2018). Perfil psicológico en una muestra de triatletas amateurs y diferencias con otros deportes. Cuadernos de Psicología de Deporte, 18(1), 55-62.

Santos, S., Sarmento, H., Alves, J., \& Campaniço, J. (2014). Construcción de un instrumento para la observación y el análisis de las interacciones en el waterpolo. Revista Psicologia Del Deporte, 23(1), 191-200.

\title{
Análise das características de jovens atletas de natação, futebol e basquetebol a nível antropométrico, de composição corporal e capacidades físicas
}

\author{
Susana Vale ${ }^{1,2}$, Tiago Venâncio ${ }^{1}$, Cátia Ferreira ${ }^{2,3}$, Teresa Figueiredo ${ }^{1,4}$, Aldo M. Costa ${ }^{5,6,7}$, \\ Mário Espada ${ }^{1,2,8}$ \\ 1. Escola Superior de Educação, Instituto Politécnico de Setúbal, Portugal; 2. Instituto Piaget, ISEIT, Campus Universitário \\ Almada, Portugal; 3. Grupo de Optimización del Entrenamiento y Rendimiento Deportivo, Universidad de Extremadura, \\ Espanha; 4. Centro de Investigação em Qualidade de Vida, Instituto Politécnico de Santarém, Portugal; 5. Departamento de \\ Ciências do Desporto da Universidade da Beira Interior, Covilhã, Portugal; 6. Centro de Investigação em Desporto, Saúde e \\ Desenvolvimento Humano, CIDESD, Vila Real, Portugal; 7. Centro de Investigação em Ciências da Saúde, Universidade da Beira \\ Interior, Covilhã, Portugal; 8. Centro Interdisciplinar de Estudo da Performance Humana, FMH, Portugal
}

\section{INTRODUÇÃO}

As características dos atletas de diferentes modalidades desportivas contribuem de forma determinante para o sucesso ao nível do desempenho, sendo o treino de força uma prática comum na maioria dos desportos, objetivando simultaneamente a melhoria do desempenho e a prevenção de lesões (Barbosa et al., 2013). Dados 
antropométricos, composição corporal, força e flexibilidade têm sido indicados como associados ao desempenho desportivo, contudo, as ações motoras e gestos técnicos diferem entre modalidades e nesse sentido torna-se importante caracterizar o perfil dos atletas de diferentes modalidades desportivas com o objetivo de melhorar o desempenho e também detetar talento em escalões etários mais baixos. O objetivo do presente estudo foi analisar as características de jovens atletas de natação, futebol e basquetebol.

\section{MÉTODOS}

Um total de 30 atletas masculinos de clubes de nível regional foram avaliados, divididos em três grupos de 10 jovens por modalidade (natação, futebol e basquetebol). Os praticantes de natação $(15.8 \pm 1.4$ anos idade, $60.8 \pm 7.5 \mathrm{~kg}$ peso, $1.72 \pm 0.06 \mathrm{~m}$ altura) de futebol $(15.1 \pm 0.3$ anos idade, $61.3 \pm 5.9 \mathrm{~kg}$ peso, $1.74 \pm 0.04 \mathrm{~m}$ altura) e basquetebol (14.9 \pm 0.3 anos idade, $62.3 \pm 7.9 \mathrm{~kg}$ peso, $1.75 \pm 0.03 \mathrm{~m}$ altura) realizaram o salto em contramovimento (SCM) na plataforma Ergojump, foram avaliados ao nível de composição corporal com balança de bioimpedância, realizaram também teste de força de preensão manual (FPM) com recurso a handgrip, e teste de flexibilidade utilizam-se o teste sit and reach executado com pés juntos. Foi utilizado o teste de Shapiro-Wilk para verificação da distribuição. Para comparação entre grupos foi utilizado o teste $t$ de amostras emparelhadas $(\mathrm{p} \leq 0.05)$.

\section{RESULTADOS}

Os nadadores foram os únicos a evidenciar valores de flexibilidade positivos $(2.40 \pm 9.55$ $\mathrm{cm})$, seguidos dos futebolistas $(-0.10 \pm 7.29 \mathrm{~cm})$ e basquetebolistas $(-0.20 \pm 3.39 \mathrm{~cm})$. Por outro lado, no SCM, os basquetebolistas evidenciaram os melhores valores $(38.98 \pm 5.19 \mathrm{~cm})$ seguido de futebolistas e nadadores com valores muitos próximos (respetivamente $35.39 \pm 2.67$ e $35.31 \pm 5.48 \mathrm{~cm}$ ). Já no que concerne a FPM, os melhores valores foram obtidos pelos futebolistas $(37.09 \pm 9.86 \mathrm{~kg})$, seguidos de basquetebolistas $(35.74 \pm 6.79)$ e nadadores $(35.10 \pm 7.84)$. Ao nível de todas as capacidades físicas avaliadas, composição corporal (massa gorda, massa muscular e percentagem de água), peso e altura, embora tenham sido observadas diferenças entre modalidades, as mesmas não foram estatisticamente significativas. Verificaram-se apenas correlações entre percentagem de massa muscular e desempenho na FPM e SCM nos nadadores (respetivamente $\mathrm{r}=0.71 ; \mathrm{p}<0.05 \mathrm{e}$ $\mathrm{r}=0.83 ; \mathrm{p}<0.01$ ) e percentagem de massa muscular e FPM nos jogadores de basquetebol $(r=0.67 ; p<0.05)$.

\section{DISCUSSÃO}

Verificou-se no presente estudo a importância de ser realizada avaliação e controlo do treino nas diferentes modalidades desportivas em função de serem observadas diferenças que se relacionam com a especificidade das práticas quotidianas, como no caso da flexibilidade na natação e salto em contramovimento no basquetebol. Outro aspeto que nos parece relevante é o facto das características entre atletas de diferentes modalidades apresentar diferenças não estatisticamente significativas, o que atribuímos ao facto da faixa etária se situar no momento imediatamente pós e durante o salto pubertário (em redor dos 15 anos de idade) onde decorrem diversas alterações a nível físico nos atletas em função do seu desenvolvimento biológico (Moreira et al., 2014). Também o nível dos atletas entendemos estar relacionado com os resultados, não sendo os jovens de nível nacional ou estando integrados no alto rendimento apresentam características semelhantes, o que é um fator a considerar no treino diariamente. É fundamental caracterizar os atletas de diferentes modalidades desportivas no sentido de otimizar o processo de treino e sua especificidade, procurando, em simultâneo, prevenir lesões e, naturalmente, melhorar o desempenho desportivo.

\section{REFERÊNCIAS}

Barbosa, T. M., Costa, M., \& Marinho, D. A. (2013). Proposal of a deterministic model to explain swimming performance. Int J Swimming Kinetics, 2, 1-54.

Moreira, M. F., Morais, J. E., Marinho, D. A., Silva, A. J., Barbosa, T. M., \& Costa, M. J. (2014). Growth influences biomechanical profile of talented swimmers during the summer break. Sports Biomech, 13(1), 62-74. 


\section{Comparação da resposta do consumo de oxigénio entre duas tarefas de treino intervalado em natação - 8X100 vs 4X200 metros}

Tiago F. Almeida1,2, Dalton M. Pessoa Filho², Astor R. Simionato², Mário C. Espada1,3, Joana F. Reis ${ }^{1,4}$, Francisco B. Alves ${ }^{1}$

1. CIPER, BIOLAD, Faculdade de Motricidade Humana; 2. UNESP, LABOREH, Rio Claro, Brasil; 3. Escola Superior de Educação, Instituto Politécnico de Setúbal; 4. Universidade Europeia, Lisboa, Portugal

\section{INTRODUÇÃO}

$\mathrm{O}$ treino intervalado $(\mathrm{TI})$ realizado à velocidade associada ao consumo máximo de oxigénio $\left(\mathrm{VO}_{2} \max \right)$ tem vindo a ser proposto como um meio efetivo na melhoria do desempenho aeróbio de atletas, sendo o tempo despendido com consumos de oxigénio $\left(\mathrm{VO}_{2}\right)$ perto dos valores máximos, um dos principais fatores que contribuem para o desenvolvimento do $\mathrm{VO}_{2} \mathrm{max}$ e desempenho desportivo (Libicz et al., 2005). O objetivo do presente estudo foi analisar a resposta do $\mathrm{VO}_{2}$ em diferentes tarefas de TI em natação e aferir se esta se relaciona com o desempenho desportivo em provas de meia distância.

\section{MÉTODOS}

Treze nadadores bem treinados realizaram um teste incremental descontínuo composto por 6 patamares de $250 \mathrm{~m}$ e uma última repetição de $200 \mathrm{~m}$ à máxima intensidade no estilo de crol (Espada et al., 2015) para obtenção do $\mathrm{VO}_{2} \max \mathrm{e}$ da velocidade aeróbia máxima (VAM). Em dias diferentes e por ordem aleatória realizaram duas séries, no mesmo estilo de nado, de TI à VAM: (a) $8 \times 100$ e (b) $4 \times 200 \mathrm{~m}$ com 15 e 30 s de intervalo, respetivamente. Para cada TI foram calculados a média do VO2max obtido em cada repetição da série $\left(\mathrm{TIMVO}_{2} \mathrm{max}\right)$, o consumo máximo da série $\left(\mathrm{TIVO}_{2} \mathrm{max}\right)$ e o tempo acima de $90 \%$ do $\mathrm{VO}_{2} \max$. A primeira repetição de cada TI serviu para o cálculo dos parâmetros da cinética do $\mathrm{VO}_{2}$ $\left(\mathrm{VO}_{2} \mathrm{~K}\right)$. Foi sempre utilizado um analisador de gases (K4b2, Cosmed, Itália), acoplado ao atleta através de um snorkel para análise dos gases expirados ao longo de todo o percurso de nado e um pacer luminoso subaquático para controlo das intensidades. A perceção subjetiva do esforço (PSE) foi registada em cada TI.

\section{RESULTADOS}

A resposta do $\mathrm{VO}_{2}$ foi muito similar entre os dois TI, com os parâmetros da $\mathrm{VO}_{2} \mathrm{~K}$, o $\mathrm{TIMVO}_{2}$ max, o $\mathrm{TIVO}_{2} \mathrm{max}$, o tempo acima dos $90 \%$ do $\mathrm{VO}_{2}$ max e o respetivo percentual para o tempo total da tarefa, a não evidenciarem diferenças estatísticas significativas entre os TI. O $\mathrm{TIVO}_{2} \mathrm{max}$ e $\mathrm{O} \quad \mathrm{TIMVO}_{2} \mathrm{max}$ apresentaram correlações entre os TI $(r=0.72, \quad p<0.01$ e $\mathrm{r}=0.70, \mathrm{p}<0.01$, respetivamente) e revelaram-se inversamente associados com o desempenho na prova dos $400 \mathrm{~m}$ livres $(\mathrm{r}=-0.57, \mathrm{p}<0.05$ e $\mathrm{r}=$ $0.67, \mathrm{p}<0.05$, respetivamente para o TI8x100; e $\mathrm{r}=-0,67, \quad \mathrm{p}<0.05$ e $\mathrm{r}=-0.59, \quad \mathrm{p}<0.05$, respetivamente para o $\mathrm{TI} 4 \times 200)$. A $\mathrm{VO}_{2} \mathrm{~K}$, através da constante temporal primária, também se revelou correlacionada com ambos os TI $(r=$ 0.73, $\mathrm{p}<0.01$ ). A PSE foi a única variável a evidenciar diferenças estatisticamente significativas sendo superior no TI4x200.

\section{DISCUSSÃO}

Independentemente da divisão de uma tarefa de $800 \mathrm{~m}$ em repetições de 100 ou 200 m, com 15 e $30 \mathrm{~s}$ de intervalo (respetivamente), a resposta do $\mathrm{VO}_{2}$ é muito similar não sendo observadas diferenças significativas que nos indiquem claramente qual o melhor TI para a melhoria do desempenho aeróbio, no entanto, os resultados da PSE sugerem a preferência dos atletas pelo TI8x100. As correlações observadas entre as variáveis sugerem que os nadadores mais eficientes do ponto de vista fisiológico (valores mais baixos de constante temporal primária e valores relativos de $\mathrm{VO}_{2}$ mais elevados) no TI8x100, são os que apresentam melhor desempenho no TI $4 \times 200$, sendo que aqueles que conseguem nadar as séries com valores superiores de $\mathrm{VO}_{2}$ são os que apresentam melhores desempenhos desportivos na prova dos $400 \mathrm{~m}$ livres.

\section{REFERÊNCIAS}

Libicz, S., Roels, B., \& Millet, G. P. (2005). VO2 responses to intermittent swimming sets at velocity associated with VO2max. Canadian Journal of Applied Physiology, 30(5), 543-553.

Espada, M., Reis, J., Almeida, T., Bruno, P., Vleck, V., \& Alves, F. (2015). Ventilatory and Physiological Responses in swimmer below and above their maximal lactate steady state. Journal of Strength $\mathcal{E}$ Conditioning Research 29(10), 2836-43. 


\title{
Relação entre desempenho nos 50, 100 e 200 m de nado crol e variáveis de força fora de água em nadadores do escalão infantis
}

\author{
Tiago Venâncio ${ }^{1}$, Pedro Polidoํㅜ, Pedro Nascimento ${ }^{1}$, Cátia Ferreira ${ }^{2,3}$, Teresa Figueiredo1,4, \\ Aldo M. Costa ${ }^{5,6,7}$, Mário Espada1,2,8 \\ 1. Escola Superior de Educação, Instituto Politécnico de Setúbal, Portugal; 2. Instituto Piaget, ISEIT, Campus Universitário \\ Almada, Portugal; 3. Grupo de Optimización del Entrenamiento y Rendimiento Deportivo, Universidad de Extremadura, \\ Espanha; 4. Centro de Investigação em Qualidade de Vida, Instituto Politécnico de Santarém, Portugal; 5. Departamento de \\ Ciências do Desporto, Universidade da Beira Interior, Covilhã, Portugal; 6. Centro de Investigação em Desporto, Saúde e \\ Desenvolvimento Humano, UTAD, Vila Real, Portugal; 7. Centro de Investigação em Ciências da Saúde, Universidade da Beira \\ Interior, Covilhã, Portugal; 8. Centro Interdisciplinar de Estudo da Performance Humana, FMH, Portugal
}

\section{INTRODUÇÃO}

O sucesso no desempenho na natação depende de um conjunto amplo de fatores onde se incluem capacidades físicas como a força dos membros inferiors e superiores. Contudo, em jovens esta capacidade física naturalmente não se encontra muito desenvolvida, o que não significa que não seja relevante decorrer avaliação e controlo do treino. Previamente Marinho et al (2011) indicaram que o treino e competição em jovens nadadores devem ser monitorizados numa base regular com o objetivo de serem definidas as apropriadas tarefas de treino e a melhoria do desempenho. A investigação com jovens nadadores é reduzida comparativamente a atletas mais velhos e experientes, o objetivo do presente estudo foi analisar a relação entre desempenho nos 50, 100 e $200 \mathrm{~m}$ de nado crol e variáveis de força fora de água em nadadores do escalão infantis.

\section{MÉTODOS}

Doze nadadores infantis (10 masculinos e 2 femininos; $13.1 \pm 0.7$ anos idade, $50.8 \pm 4.8 \mathrm{~kg}$ peso, $1.62 \pm 0.02 \mathrm{~m}$ altura) realizaram um conjunto de testes dentro e fora de água. $\mathrm{Na}$ água, com partida de baixo dos blocos, 50, 100 e $200 \mathrm{~m}$ máximos de nado crol (T50, T100 e T200). No ginásio, teste do salto em contramovimento (SCM) na plataforma Ergojump, lançamento da bola medicinal (LBM) de $3 \mathrm{~kg}$ e análise de composição corporal com balança de bioimpedânica. Foi utilizado o teste de ShapiroWilk para verificação da distribuição e aceite significância estatística a $\mathrm{p} \leq 0.05$.

\section{RESULTADOS}

$\mathrm{T}_{50}, \mathrm{~T}_{100}$ e $\mathrm{T}_{200}$ (respetivamente, $32.3 \pm 2.38$, $71.54 \pm 5.48$ e $158.85 \pm 12.37$ segundos) revelaram-se naturalmente correlacionados entre si, mas com maior expressão entre $T_{100}$ e $T_{200}$ $(\mathrm{r}=0.84 ; \mathrm{p}<0.01)$, comparativamente a $\mathrm{T}_{50}$ e $\mathrm{T}_{200}$ $(\mathrm{r}=0.82 ; \mathrm{p}<0.01)$ e $\mathrm{T}_{50}$ e $\mathrm{T}_{100}(\mathrm{r}=0.72 ; \mathrm{p}<0.01)$. O LBM $(3.59 \pm 0.40 \mathrm{~m})$ observou-se como mais correlacionado com a maior distância de nado, $\mathrm{T}_{200}(\mathrm{r}=-0.80 ; \mathrm{p}<0.01)$, mas também com $\mathrm{T}_{100}$ $(\mathrm{r}=-0.76 ; \mathrm{p}<0.01)$ e $\mathrm{T}_{50}(\mathrm{r}=0.74 ; \mathrm{p}<0.01)$. Já o SCM $(30.33 \pm 4.74 \mathrm{~cm})$ foi observado como correlacionado apenas com $\mathrm{T}_{50} \quad(\mathrm{r}=-0.67$; $\mathrm{p}<0.05)$. A altura correlacionou-se com $\mathrm{T}_{200}(\mathrm{r}=-$ $0.69 ; \mathrm{p}<0.05)$ e o LBM $(\mathrm{r}=0.64 ; \mathrm{p}<0.05)$. Ao nível de composição corporal (massa gorda, massa muscular e percentagem de água) não foram observadas correlações com o desempenho nos testes dentro e fora de água.

\section{DISCUSSÃO}

Este estudo confirma que as correlações entre LBM e distância de nado têm tendência a diminuir com o aumento da distância de nado, facto previamente verificado por Garrido et al. (2010) na análise realizada entre os 25 e $50 \mathrm{~m}$, e agora confirmado com a análise de distância de nado superiores. Verifica-se assim em jovens nadadores uma maior importância no desempenho de nado em função da aplicação de força na água dos membros superiores comparativamente a membros inferiores, altura e composição corporal, aspeto que entendemos naturalmente relacionar-se com a idade dos jovens atletas. Por outro lado, a relação entre distâncias de nado evidencia que devem ser priviligiadas distância de treino e provas de 50 e $200 \mathrm{~m}$ nestas idades no sentido de melhoria de aspetos relacionados com a velocidade (na distância mais reduzida) e aptidão aeróbia nos $200 \mathrm{~m}$, uma vez que do ponto de vista fisiológico a distância de $100 \mathrm{~m}$ não permite uma aproximação ao consumo máximo de oxigénio por parte dos atletas.

\section{REFERÊNCIAS}

Garrido, N., Marinho, D. A., Barbosa, T. M., Costa, A. M, Silva, A. J., Pérez-Turpin, J. A., \& Marques, M. C. (2010). Relationship Between Dry Land 
Strength, Power Variables and Short Sprint Performance in Young Competitive Swimmers. Journal of Human Sport and Exercise, 5(2), 240-249. Marinho, D. A., Amorim, R. A., Costa, A. M., Marques,
Anaerobic critical velocity and swimming performance in young swimmers. Journal of Human Sport and Exercise, 6, 80-86.

\section{Impacto do treino de força no tempo de partida em jovens nadadores}

Tomás Capucho ${ }^{1}$, Paulo N. Vieira ${ }^{1}$, Ricardo Antunes ${ }^{1}$, Nuno Santos ${ }^{2}$, Joana Reis ${ }^{1}$

1. Universidade Europeia, Laureate International Universities; 2. GESLOURES, Loures, Portugal

\section{INTRODUÇÃo}

O tempo de partida parece estar associado com a força e potência dos membros inferiores dos nadadores(West et al., 2011). O objectivo deste estudo foi estudar o efeito de oito semanas de treino de força com exercícios gerais calisténicos na força inferior e no tempo de partida em nadadores jovens.

\section{MÉTODOS}

Doze nadadores jovens (seis do sexo feminino, $12,5 \pm 0.9$ anos; $158,7 \pm 7,3 \mathrm{cms} ; 48,3$ $\pm 4,9 \mathrm{~kg}$ ) foram divididos aleatoriamente em 2 grupos: Treino de Força (TF) e Controlo (C). Durante oito semanas, ambos os grupos realizaram o mesmo treino de água (cinco sessões semanais). Adicionalmente, o grupo TF realizou 2 sessões semanais de treino de força em seco, constituído por exercícios gerais calisténicos com periodização linear. Antes e após o processo de treino, os nadadores realizaram avaliações de força inferior através do Squat-Jump (SJ) e Countermovement Jump (CMJ) (Chronojump Bosco systems, Barcelona, Espanha) e foram registados os tempos de partida aos 5 e 15 metros. Foi aplicado o teste Mann-Whitney para comparar o impacto do treino nos dois grupos. Assumiu-se valor de significância $p \leq 0.05$.

\section{RESULTADOS}

O grupo TF apresentou um aumento superior no valor de Potência relativa no SJ. Nas variáveis obtidas no CMJ e tempo de partida (T5 e T15), não existiram diferenças significativas entre grupos.

Tabela 1

Comparação entre grupos

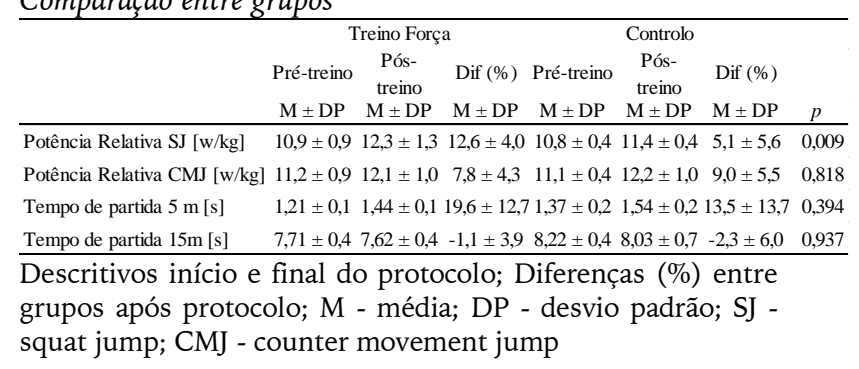

\section{DISCUSSÃO}

Apesar dos nadadores que realizaram treino de força em seco terem apresentado ganhos superiores na força concêntrica de membros inferiores, esta melhoria não se refletiu na performance do tempo de partida. Este facto pode estar associado à pouca estimulação da força reactiva no processo de treino, que pode estar associada à ausência de diferenças entre os dois grupos na potência relativa do CMJ. Em nadadores jovens, para além do impulso no bloco de partida, outros fatores técnicos poderão ser determinantes no desempenho da partida.

\section{REFERÊNCIAS}

West, D. J., Owen, N. J., Cunningham, D. J., Cook, C. J., \& Kilduff, L. P. (2011). Strength and power predictors of swimming starts in international sprint swimmers. Journal of strength \& Conditioning Research, 25(4), 950-5. 


\section{A influência de um ciclo de treino da força em curtas distâncias de nado}

Tiago J. Lopes ${ }^{1}$, Carlota A. Gonçalves ${ }^{1}$, Henrique P. Neiva1,2, Daniel A. Marinho', ${ }^{1,2}$

1. Departamento de Ciências do Desporto da Universidade da Beira Interior, Covilhã, Portugal; 2. Centro de Investigação em Desporto, Saúde e Desenvolvimento Humano, CIDESD, Vila Real, Portugal

\section{INTRODUÇÃo}

Variáveis associadas ao treino de força revelam-se como indicadores de performance em curtas distâncias na natação. Um programa específico de treino de força (TF) poderá aumentar a taxa de produção de força, resultando em melhorias na performance (Marques et al., 2011). O presente estudo pretende avaliar a influência do TF na melhoria da performance de nado (PN) nos 50 e $100 \mathrm{~m}$ livres.

\section{MÉTODOS}

Catorze nadadores masculinos (idade: 20.57 \pm 1.79 anos; massa corporal: $72.14 \pm 5.78 \mathrm{~kg}$; altura: $179.86 \pm 4.96 \mathrm{~cm})$ participaram no estudo. O grupo experimental (GE) foi sujeito a um TF de 8 semanas, que complementou o treino de água, enquanto que o grupo de controlo (GC) foi somente sujeito ao treino de água. A performance foi avaliada nos 50 e $100 \mathrm{~m}$ livres, enquanto que a força foi avaliada no agachamento (SQ), supino reto (BP), salto com contramovimento $(\mathrm{CMJ})$ e com mãos livres (CMJFA) e lançamento de bola medicinal (MBT). Para comparar as diferenças entre os dois períodos de avaliação (M1 e M2) entre os dois grupos, utilizou-se a ANOVA bidirecional, com medidas repetidas com um fator (momento de avaliação), considerando as variáveis em estudo.

\section{RESULTADOS}

Na Tabela 1 é apresentada uma interação significativa em MBT e moderada nas restantes variáveis analisadas quando se compara pré vs pós treino em cada grupo. No desempenho de nado a interação (grupo vs. momento) foi significativa no $2^{\circ}$ parcial de $50 \mathrm{~m}$ nos $100 \mathrm{~m}\left(p<0.01 ; \mathrm{n}_{\mathrm{p}}{ }^{2}=0.50\right)$, no tempo de $100 \mathrm{~m}\left(p<0.05 ; \mathrm{n}_{\mathrm{p}}^{2}=0.42\right)$, e no $1^{\circ}$ parcial aos $25 \mathrm{~m}$ nos $50 \mathrm{~m}\left(p<0.01 ; \mathrm{n}_{\mathrm{p}}{ }^{2}=0.29\right)$.

Tabela 1

Comparação entre os dois grupos no desempenho no treino de força em seco (TF).

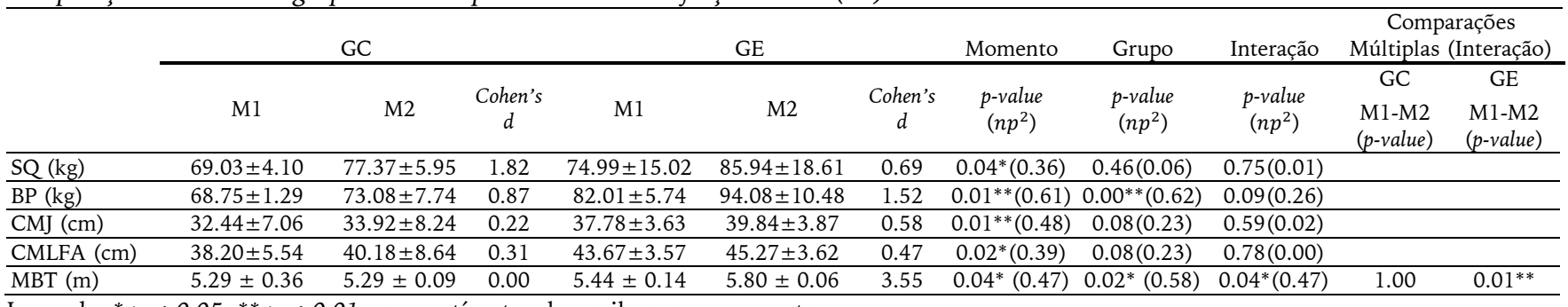

Legenda: ${ }^{*} p<0.05,{ }^{* *} p<0.01 ; \mathrm{cm}$-centímetro; kg-quilogramas; m-metros.

\section{DISCUSSÃO E CONCLUSÃO}

Os resultados demonstram que os indicadores de TF estão associados à melhoria do desempenho de sprint durante o nado.

\section{REFERÊNCIAS}

Marques, M. C., Zajac, A., Pereira, A., \&Costa, A. M. (2011). Strength training and detraining in different populations: Case Studies. Journal of Human Kinetics, 7-14.

\section{The two methods of teaching swimming in hungarian schools}

\section{Zsófia Kovács ${ }^{1}$, Ferenc Tóvári², Gyöngyvér Prisztóka ${ }^{3}$, Attila Kajos ${ }^{4}$}

1. Education and Society Doctoral School of Education PTE BTK, Hungary; 2. Department of Individual Sports, PTE TTK Institute of Sport Sciences an Physical Education, Hungary; 3. Head of Department of Sports Theory and Management, PTE TTK Institute of Sport Sciences an Physical Education, Hungary; 4. Department of Sports Theory and Management, PTE TTK Institute of Sport Sciences an Physical Education, Hungary

\section{INTRODUCTION}

Swimming, wich is also known for a life saving function, has many important effects justifying that every child should be able to learn the basics of this sport in a school setting. In our country the „Every child should learn to swim!” program 
promoted by the everyday PE and the World Aquatics Championship, introduced in 2013, is providing broader opportunities for having swimming instruction in a school setting.

\section{GOAL}

The goal of our work is to show the application and efficiency in a school setting by comparing two swimming instruction methods all in conjuction with the optimal timeframe that can be spent on teaching.

\section{MATERIAL METHODS}

We tested the swimming skills $(n=474)$ coming from two county seats (Pécs, Kaposvár) one-by-one. I processed the resulting data with simple mathematical-statistical methods (average and percentage calculation) using Microsoft Excel ${ }^{\circledR}$ software.

\section{RESULTS}

Based on our results the fact that the majority of students learn the basics of different swimming moves in the lessons provided by the school's time frame can be proved. However, it is not enough to reach the ability for continuous swimming, or to be recommended for water sports.

\section{REFERENCES}

Magyar Úszó Szövetség (2013). Minden gyermek tanuljon meg úszni ["Every child should learn to swim!"]. Budapest: MÚSZ.

Tóth, Á., Sós, Cs., \& Egressy, J. (2007). Úszás edzésmódszertan [Swimming training methodology]. Budapest: Viva Média Holding. 